\title{
Pathogenese und Therapie der Akne: Aktuelle Aspekte
}

\section{Pathogenesis and Therapy of Acne: Current Aspects}

\author{
Autor \\ F. R. Ochsendorf \\ Institut \\ Zentrum Dermatologie und Venerologie, Klinikum der J. W. Goethe-Universität Frankfurt/M.
}

\section{Bibliografie}

Dol $10.1055 / \mathrm{s}-2007-966124$

Akt Dermatol 2007; 33;

137-141 @ Georg Thieme

Verlag KG Stuttgart · New York ISSN 0340-2541

Korrespondenzadresse

Prof. Dr. Falk R. Ochsendorf Zentrum Dermatologie und Venerologie Klinikum der J. W. GoetheUniversität

Theodor-Stern-Kai 7 60590 Fankfurt/M. ochsendorf@em.unifrankfurt.de

\section{Zusammenfassung \\ $\nabla$}

Acne vulgaris ist eine Erkrankung mit einer Prävalenz bei Jugendlichen bis zu $80 \%$. Genetische Faktoren spielen eine große Rolle. Verursacht wird sie durch ein komplexes Zusammenspiel verschiedener Faktoren (Seborrhoe, Verhornungsstörung, mikrobielle Besiedelung mit Propionibacterium acnes sowie Entzündungsreaktionen). Entzündungsreaktionen sind offenbar schon bei der Entstehung früher Akneläsionen, den Mikrokomedonen, beteiligt. Je nach klinischer Ausprägung können die einzelnen pathogenetischen Faktoren effektiv behandelt werden:

\section{Einleitung}

\section{$\nabla$}

Die Akne hat im jugendlichen Alter eine Prävalenz von bis zu 80\% [1]. Die typischen klinischen Veränderungen der Akne, d.h. seborrhoische Haut, Komedonen, Papeln, Pusteln, Knoten und Zysten, sind auch Nicht-Dermatologen gut bekannt. In den letzten Jahren haben sich die Kenntnisse der Pathogenese dieser Erkrankung deutlich erweitert. Es stehen zahlreiche sehr gut wirksame Behandlungsmöglichkeiten der verschiedenen Akneformen zur Verfügung, so dass die Akne nicht einfach nihilistisch ertragen werden muss. Im Folgenden werden aktuelle pathogenetische und therapeutische Aspekte zusammengefasst.

\section{Pathogenese \\ $\nabla$}

Die Acne vulgaris entsteht im Talgdrüsenfollikel durch ein komplexes Zusammenspiel von starker Seborrhoe, einer Verhornungsstörung im Sinne einer Retentions- und Proliferationshyperkeratose im Infrainfundibulum des Talgdrüsenfollikels, einer mikrobiellen Besiedelung mit Propioni-
Verhornungsstörungen mit topischen Retinoiden und Benzoylperoxid (BPO), bakterielle Besiedlung mit topischen und systemischen Antibiotika sowie BPO, Entzündungen mit Antibiotika, die Seborrhoe mit Antiandrogenen und alle Faktoren mit Isotretinoin. In den letzten Jahren wurde die Überlegenheit von Kombinationsbehandlungen mit verschiedenen der genannten Substanzen dokumentiert, wie systemische Antibiotika und topische Retinoide oder BPO/Antibiotikum-Kombinationen. Retinoide sind die Mittel der Wahl zur Erhaltungstherapie. Mit diesen Therapieansätzen kann die Akne sehr effektiv behandelt werden.

bacterium acnes sowie Entzündungsreaktionen. Es wurde in den letzten Jahren nachgewiesen, dass diese Entzündungsreaktionen in der Pathogenese der Akne-Effloreszenzen sehr früh auftreten und offenbar schon bei der Entstehung der Mikrokomedonen beteiligt sind [2].

\section{Genetische Faktoren}

Offensichtlich spielen bei der Akne-Entstehung genetische Faktoren eine große Rolle. Dies resultiert aus der Beobachtung, dass die Größe und Aktivität der Talgdrüsen, der Seborrhoe und die Porengröße und damit die Neigung zur AkneEntwicklung vererbt wird [3]. 50\% der Patienten mit Akne nach der Pubertät hatten wenigstens einen Verwandten ersten Grades mit gleichartiger Akne [4]. Auch Verwandte von Patienten mit persistierender Akne ( $>25$ Jahre; $n=204$ ) hatten im Vergleich zu entsprechenden Kontrollen ( $n=144$ ) ein etwa 4-fach erhöhtes Risiko zu einer Persistenz der Akne (odds ratio 3,93, 95\% Konfidenz-Intervall 2,79-5,51, $\mathrm{p}<0,001$ [5]. Da gleichartige Umweltfaktoren nicht als pathogenetischer Faktor ausgeschlossen werden können, sind Zwillingsstudien aussagekräftiger. Studien verschiedener Qualität und Studiengröße kamen 


\begin{tabular}{|c|c|c|c|c|}
\hline Autor & Jahr & Zahl & Wesentliche Befunde & Schlussfolgerung \\
\hline Friedman [30] & 1984 & $\begin{array}{l}930 \text { Paare } \\
\text { (342 MZ, } \\
345 \mathrm{DZ}, 243 \\
\text { unbekannt) }\end{array}$ & & $\begin{array}{l}\text { Substanzieller genetischer } \\
\text { Einfluss }\end{array}$ \\
\hline Walton [31] & 1988 & $2 \times 20$ Paare & $\begin{array}{l}\text { MZ: Talgsekretion identisch, } \\
\text { Akne-Schwere unterschied- } \\
\text { lich } \\
\text { DZ: Talgsekretion unter- } \\
\text { schiedlich; Akne-Schwere } \\
\text { unterschiedlich }\end{array}$ & $\begin{array}{l}\text { v. a. Talgsekretion genetisch } \\
\text { determiniert }\end{array}$ \\
\hline Cunliffe [32] & 1989 & 95 Paare & $\begin{array}{l}\text { MZ: } 95 \% \text { Akne } \\
\text { DZ: } 46 \%\end{array}$ & $\begin{array}{l}\text { Substanzieller genetischer } \\
\text { Einfluss für Akne-Entstehung }\end{array}$ \\
\hline Bataille [33] & 2002 & $\begin{array}{l}458 \mathrm{MZ} \\
1099 \mathrm{DZ}\end{array}$ & $\begin{array}{l}81 \% \text { ( } 95 \% \text { Konfidenz-Intervall } \\
73-87 \% \text { ) der Varianz der } \\
\text { Schwere der Erkrankung } \\
\text { konnte durch genetische } \\
\text { Effekte erklärt werden. } \\
19 \% \text { wurden durch andere, } \\
\text { nicht gemeinsame Faktoren } \\
\text { (wie Umwelteinflüsse) erklärt }\end{array}$ & $\begin{array}{l}\text { Ca } 80 \% \text { der Akne-Schwere ist } \\
\text { genetisch bedingt }\end{array}$ \\
\hline Evans [34] & 2005 & 778 Paare & $\begin{array}{l}\text { MZ: 0,73-0,95 Akne } \\
\text { DZ: 0,14-0,64 Akne }\end{array}$ & $\begin{array}{l}\text { Schwere und Lokalisation in } \\
\text { jedem Alter stark durch } \\
\text { genetische Faktoren beein- } \\
\text { flusst }\end{array}$ \\
\hline
\end{tabular}

Tab. 1 Akne und Genetik: Zusammenstellung von Zwillingsstudien

$\mathrm{MZ}=$ monozygote, $\mathrm{DZ}=$ dizygote $Z$ willinge.

alle zum gleichen Ergebnis, dass ein hoher Anteil der AkneSchwere und -Ausprägung durch genetische Faktoren erklärt werden kann ( Tab. 1). Bisher konnte aber kein „Akne-Gen“ identifiziert werden. Gefunden wurden verschiedene genetische Polymorphismen. Es wird angenommen, dass für verschiedene Akne-Formen unterschiedliche genetische Dispositionen verantwortlich sein könnten (wie A. neonatorum, infantilis, Pubertäts-A., A. conglobata) [6]. Wenn man nach Defekten der 21-Hydroxylase sucht, findet man diese insbesondere bei Patienten mit fehlendem Ansprechen auf eine adäquate Behandlung [7 -9]. Insbesondere bei einer Akne im Kindesalter sollte an diese Möglichkeit des Vorliegens eines adrenogenitalen Syndroms gedacht werden [10].

\section{Androgene, Talgproduktion und Entzündung}

Androgene stimulieren die Talgproduktion über Androgenrezeptoren in der Talgdrüse und sind Voraussetzung für die Entwicklung einer Akne. Die Talgdrüse besitzt aber selbst alle Enzyme, um Androgene zu synthetisieren bzw. aus schwach wirksamen Vorläufern potentere Androgene zu generieren. Darüber hinaus wird die Talgproduktion aber auch über die Aktivierung von „Peroxisom Proliferator aktivierter Rezeptor“ (PPAR alpha und gamma) angeregt. Dabei ist offenbar ein über den 5-Lipoxygenase-Weg entstehender Arachidonsäure-Metabolit, das Leukotrien-B4, einer der stärksten natürlichen Liganden. Die Aktivität der Talgdrüse wird nicht nur durch Androgene, sondern auch durch regulierende Neuropeptide, u.a. Substanz P, Corticotropin-Releasing Hormon und $\mathrm{ACTH}$, moduliert. Arachidonsäure und langkettige Omega-6-Fettsäuren stimulieren die Talgdrüse zur Bildung von Lipiden und Interleukinen (Il-6, Il-8). Über das Verhältnis von anti-inflammatorischen Omega-3- und pro-inflammatorischen Omega-6-Fettsäuren (in westlicher Ernährung $1: 20$, in anderen Kulturen mit weniger Akne $1: 1$ ) könnte die Ernährung die Akne modulieren. Zwischen der Anzahl der gerauchten Zigaretten und der Schwere der Akne besteht eine Assoziation. Dies könnte durch pro-inflammatorische Substanzen im Zigarettenrauch, wie Arachidonsäure, oder durch assoziierte Ernährungsgewohnheiten erklärt werden [11].

\section{Mikrokomedo und Entzündung}

Der Mikrokomedo wird offenbar über entzündliche Prozesse, vermutlich über IL-1 alpha, induziert. IL-1 alpha wird von normalen Talgdrüsen produziert und stimuliert die Proliferation von Keratinozyten [12,13].

\section{Propionibacterium acnes und Entzündung}

Die Bedeutung von $P$. acnes und $P$. granulosum bei der Akne-Entstehung wird derzeit kontrovers diskutiert. Während manche Autoren diesem Erreger eher eine geringe Relevanz zusprechen, sehen andere durch $P$. acnes induzierte immunologische Mechanismen als relevant an. So werden durch $P$. acnes gebildete Lipasen, die Talg zu pro-inflammatorischen freien Fettsäuren spalten, sowie die Induktion der Bildung von IL-12, das eine TH-1 Zell-Antwort induziert, diskutiert. Patienten, die zu einer Vernarbung neigen, scheinen in der Spätphase der Entzündung eine spezifische Immunantwort auszubilden (ergänzende Literatur zu diesen komplexen Vorgängen finden sich in den Zitaten $[11,14,15])$.

\section{Therapie \\ $\nabla$ \\ Complianceförderung}

Voraussetzung für eine wirksame Behandlung der Akne ist die Gewinnung des Vertrauens des Patienten und die Motivation zur Behandlung. Dabei wird der Leidensdruck des Patienten vom Arzt oft überschätzt, zumal dieser nicht mit dem objektiven klinischen Bild korreliert [16]. Bei Akne-Patienten muss man davon ausgehen, dass jeder 2. bis 3. Patient die Behandlung nicht so durchführt, wie vom Arzt verordnet (Gieler, U. und Kupfer, J., persönliche Mitteilung). 


\begin{tabular}{|c|c|c|c|c|}
\hline & Talgproduktion & Komedogenese & Entzündung & $\begin{array}{l}\text { Reduktion } \\
\text { P. acnes }\end{array}$ \\
\hline \multicolumn{5}{|l|}{ Retinoide } \\
\hline Tretinoin & - & ++ & $+1-$ & - \\
\hline Isotretinoin (top.) & - & ++ & $+1-$ & - \\
\hline Adapalen & - & ++ & ++ & - \\
\hline Benzoylperoxid & - & $+1-$ & ++ & ++ \\
\hline Azelainsäure & - & + & + & + \\
\hline \multicolumn{5}{|l|}{ Antibiotika } \\
\hline Erythromycin & - & $+1-$ & ++ & ++ \\
\hline Tetrazykline & - & $+1-$ & ++ & ++ \\
\hline Clindamycin & - & $+1-$ & ++ & ++ \\
\hline Nadifloxacin & - & $+1-$ & ++ & ++ \\
\hline \multicolumn{5}{|l|}{ Kombination } \\
\hline Zink/Erythromycin & - & $+1-$ & ++ & ++ \\
\hline Tretinoin/Erythromycin & - & ++ & ++ & ++ \\
\hline Isotretinoin/Erythromycin & - & ++ & ++ & ++ \\
\hline BPO/Erythromycin & - & ++ & ++ & ++ \\
\hline BPO Clindamycin & - & ++ & ++ & ++ \\
\hline Isotretinoin & +++ & ++ & ++ & ++ \\
\hline \multicolumn{5}{|l|}{ Antiandrogene (syst.) } \\
\hline Cyproteronacetat & ++ & + & + & $+1-$ \\
\hline Drospirenon & ++ & + & + & $+1-$ \\
\hline Desogestrel & ++ & + & + & $+1-$ \\
\hline
\end{tabular}

Tab. 2 Effekte verschiedener Substanzen auf die pathogenetischen Faktoren der Akne
Um die Mitarbeit anzuregen, empfiehlt es sich, folgende Fragen im Gespräch in einer Sprache zu vermitteln, die der Patient verstehen kann: „1. Wie soll das Präparat angewendet werden?“; „2. Wie oft soll das Präparat angewendet werden?“; „3. Was ist bei Problemen zu tun?" Hilfreich ist es, dabei den Blickkontakt zu suchen und von Anfang an die Eigenverantwortlichkeit des Patienten zu steigern.

Da falsche Konzepte und Erwartungen weit verbreitet sind („Abheilung der Erkrankung nach der ersten Behandlung“), müssen diese angesprochen und durch realistische Ziele ersetzt werden. Nur so gelingt es, dass Patienten Therapien über längere Zeit konsequent anwenden und nur so lassen sich gute Therapieerfolge erreichen. Nach Daten von Cunliffe aus dem Jahre 1993 führte eine Kombination aus topischem Benzoylperoxid und systemischen Tetrazyklinen $(1 \mathrm{~g} / \mathrm{d})$ nach 3 Monaten zu einer 50\%igen und erst nach 6 Monaten zu einer 80\%igen Besserung des klinischen Befunds [17]. Nach neueren Studien soll die stärkste Besserung in den ersten 6 Wochen auftreten [18].

\section{Behandlungskonzept}

Die Behandlung sollte sich nach dem klinischen Bild richten. Die verfügbaren Präparate haben unterschiedliche Effekte auf die einzelnen pathogenetisch relevanten Faktoren ( $\bullet$ Tab.2). Es wurde ein Stufenschema vorgeschlagen. Eine A. comedonica wird mit topischen Retinoiden, eine A. papulopustulosa mit einer Kombination von Retinoiden und topischen antibakteriellen Substanzen behandelt. Bei mittelschweren Formen bzw. solchen mit größerer Ausdehnung werden orale Antibiotika mit topischen Retinoiden, BPO und/oder Antibiotikum kombiniert. Die Acne conglobata wird mit Isotretinoin behandelt [19].

Im Zentrum der Therapieansätze steht dabei der Mikrokomedo, dessen Entstehung verhindert werden soll. Bei milder bis moderater Akne im Gesicht zeigte sich, dass die topische Behandlung mit Benzoylperoxid genauso effektiv war wie eine systemische Tetrazyklin-oder Minocyklin-Therapie. Kombinationen von BPO mit topischem Erythromycin waren noch etwas wirksamer [18]. Bei umschriebener Akne ist damit primär eine topische Therapie sinnvoll. Hier stehen zahlreiche Präparate mit unterschiedlichen Angriffspunkten zur Verfügung ( $\bullet$ Tab. 2).

\section{Topische Retinoide}

Topische Retinoide wirken auf die Vorläuferläsion aller Akne-Effloreszenzen, den Mikrokomedo. Deshalb sind sie Mittel der ersten Wahl bei leichter bis mittelgradiger Akne und zur Erhaltungstherapie. Es stehen Tretinoin 0,05\% Creme/Lösung, Isotretinoin 0,05\% Gel und Adapalen 0,1\% Gel/Creme zur Verfügung. Daneben gibt es Kombinationspräparate (Tretinoin 0,025\%/ Erythromycin $4 \%$ bzw. Isotretinoin 0,05\%/Erythromyicn $2 \%$ ). Die neueren topischen Retinoide (Isotretinoin, Adapalen) irritieren die Haut weniger als Tretinoin. Zur Vermeidung ist eine sich langsam steigernde Anwendung zu empfehlen. Cremes reizen weniger als Gele. In den ersten Wochen können durch Ruptur der Mikrokomedonen entzündliche Veränderungen zunehmen (am geringsten bei Adapalen). Obwohl eine relevante systemische Absorption nicht nachgewiesen wurde, sollten Frauen darauf hingewiesen werden, nicht schwanger zu werden bzw. das Präparat bei Eintritt einer Schwangerschaft abzusetzen.

Retinoide haben einen festen Platz bei der Erhaltungstherapie. In einer aktuellen kontrollierten Studie wurde nachgewiesen, dass nach einer erfolgreichen Behandlung mit Minocyclin und topischem Retinoid (in diesem Fall Tazaroten) über 3 Monate kein signifikanter Unterschied gefunden wurde zwischen einer Weiterbehandlung mit dem topischen Retinoid allein, dem Antibiotikum allein oder einer Kombination von beiden [20]. Ähnliche Befunde fanden sich für Adapalen: Nach einer 3-monatigen Therapie mit Doxycyclin (mit oder ohne Adapalen) wurde für weitere 16 Wochen mit Plazebo oder Adapalen weiterbehandelt. Die Adapalen-Therapie führte zu signifikant besseren Erhaltungsraten (75 gegen 54\%) [21].

\section{Benzoylperoxid (BPO)}

Dies steht als 2,5\%, 3\%, 5\%, 10\% Gel, Creme oder Waschlotion (on/off Präparationen) zur Verfügung. Höhere Konzentrationen sind nicht wirksamer, irritieren aber mehr. Eine $2 \times$ tägliche BPO- 
Anwendung war gleich wirksam wie eine systemische antibiotische Therapie (leichte bis moderate Akne des Gesichts). Im Gegensatz zu topischen oder systemischen Antibiotika beeinflusst die bakterielle Resistenzsituation die Therapieerfolge bei BPOAnwendung nicht [18]. Die Kombination von BPO und Erythromycin war in dieser Studie etwas wirksamer. Allergische Reaktionen sind sehr selten. Patienten müssen über die bleichende Wirkung von BPO aufgeklärt werden. BPO kann während der Schwangerschaft verwendet werden.

\section{Azelainsäure}

Verfügbar als $15 \%$ Gel und 20\% Creme wirkt die Substanz auf die Entwicklung von Mikrokomedonen und vermindert entzündliche Läsionen. Die irritierende Wirkung ist geringer als die von BPO. Die Substanz kann während einer Schwangerschaft verwendet werden.

\section{Topische Antibiotika}

Verfügbar sind Erythromycin (2\% und 4\%), Clindamycin (1\%) und Tetrazyklin (3\%) als Lösung, Gel, Emulsion und Salbe. Studienvergleiche zeigten, dass Erythromycin und Clindamycin gleich wirksam waren wie BPO und dass topisches Tetrazyklin am wenigsten effektiv war. Die Wirksamkeit von Erythromycin wird bei einer Resistenz von $P$. acnes verschlechtert. $\mathrm{Zu}$ deren Prophylaxe ist eine Kombination mit BPO sinnvoll. Kombinationen (fix oder Anwendung zu verschiedenen Tageszeiten) von Antibiotika mit Retinoiden oder BPO wirkten synergistisch. Eine fixe BPO/Clindamycin Kombination muss nur einmal täglich verwendet werden und war in der Kombination wirksamer als die Einzelkomponenten [22]. Kürzlich wurde auch Nadifloxacin (1\%) als Creme zur Behandlung der Akne zugelassen. Es war gleich wirksam wie ein $2 \%$ Erythromycin-Gel [23].

\section{Systemische Antibiotika}

Gegenüber Plazebo wirken systemisch applizierte TetrazyklinAntibiotika (Tetrazyklin $1000 \mathrm{mg} / \mathrm{d}$, Doxycyclin 100(-200) mg/d, Minocyclin 100(-200) mg/d, Lymecyclin 300(-600) $\mathrm{mg} / \mathrm{d}$ und Erythromycin $1000 \mathrm{mg} / \mathrm{d}$ signifikant stärker bei der Behandlung der entzündlichen Akne. Ähnlich gut wirksam ist Clindamycin. Eindeutige Unterschiede zwischen den verschiedenen Substanzen innerhalb der Gruppe der Tetrazykline bzw. Tetrazyklin/Erythromycin lassen sich nicht ableiten. Die Daten zur Kombination mit topischen Mitteln sprechen für synergistische Effekte (topisch BPO bzw. Retinoide), so dass systemische Antibiotika nicht als Monotherapie verordnet werden sollten. Bei gleicher Wirksamkeit sind zur Auswahl weitere Kriterien, wie Pharmakokinetik, die Nebenwirkungsrate, die Resistenzentwicklung und die Behandlungskosten zu berücksichtigen. Die Therapie sollte bis zu 3 Monaten betragen; längere Behandlungen sollten mit BPO zur Resistenzverminderung kombiniert werden Es sollte zur Verhinderung von Resistenzen keine Kombination mit topischen Antibiotika einer anderen Klasse erfolgen. [24].

\section{Antibiotika-Resistenz}

Mehrere Studien belegen eine abnehmende Wirksamkeit oraler und topischer Antibiotika mit zunehmender Resistenz von $P$. acnes [18]. Deshalb sollte auf eine antibiotische Monotherapie verzichtet werden, nur so lange behandelt werden, bis keine Besserung mehr eintritt, auf eine strenge Compliance geachtet werden (s.o.), lokale und systemische Antibiotika nicht kombiniert werden sowie auf einen Wechsel der Antibiotikaklasse bei erneuter systemischer Therapie verzichtet werden. Vor allem aber sollte die Behandlung mit BPO kombiniert werden. Dies kann in fixer Kombination, im Wechsel oder im Intervall (für $>2$ Tage) erfolgen. Antibiotika sollten nicht zur Erhaltungstherapie eingesetzt werden [25].

\section{Antiandrogene}

Antiandrogene vermindern die Talgproduktion und damit indirekt die Komedogenese. Sie erwiesen sich als wirksam bei nichtentzündlicher und entzündlicher Gesichtsakne. Bei Frauen mit Zeichen einer Hyperandrogenämie sind sie Mittel der Wahl. Am stärksten wirksam ist Cyproteronacetat, gefolgt von Dienogest [26].

\section{Isotretinoin}

Die Verordnung von Isotretinoin zur Behandlung der Akne ist nach den neuen Richtlinien der europäischen Arzneimittelbehörde nur bei Personen > 12 J. mit schwerer Akne (nodulär, conglobata) möglich, die nicht auf eine adäquate kombinierte antibiotische/topische Therapie ansprechen. Die Anfangsdosis sollte bei $0,5 \mathrm{mg} / \mathrm{kg}$ KG liegen. Auf strenge Kontrazeption ist zu achten: Vor Beginn der Behandlung muss ein negativer Schwangerschaftstest vorliegen und dieser muss alle 4 Wochen während der Therapie und bis 5 Wochen nach Therapieende kontrolliert werden. Es muss mindestens eine, besser 2 (davon eine Barrieremethode) Kontrazeptionsmethode angewendet werden. Leberund Lipidwerte müssen vor, 1 Monat nach Therapiebeginn und dann alle 3 Monate kontrolliert werden. Gleichzeitige Anwendung von Peeling, Wachsepilation und Laserbehandlungen sollten bis 6 Monate nach Therapieende vermieden werden. Kombination mit Erythromyin ist möglich, mit Tetrazyklinen wegen möglicher intrakranialer Hypertension zu vermeiden. Zur Verbesserung der Resorption muss auf die Einnahme mit einer fetthaltigen Mahlzeit hingewiesen werden. Es können jeweils nur 30 Tabletten verordnet werden und das Rezept ist nur 7 Tage gültig. Auch wenn die Datenlage eher gegen die Auslösung von Stimmungsschwankungen, Depression und Suizidalität spricht (das Vorhandensein von Akne ist mit Angst und Depression assoziiert!), sind individuelle idiosynkratische Reaktionen nicht auszuschließen. Deshalb sollte diese Möglichkeit mit dem Patienten besprochen werden, damit er dies mit seiner Familie/ Freunden thematisieren kann, um entsprechende Reaktionen frühzeitig zu entdecken [27].

\section{Ergänzende Verfahren}

Neben den genannten stehen weitere Therapie-Optionen zur Verfügung: Kortikosteroide für Acne fulminans, Dapson mit antientzündlicher Wirkung in bestimmten Indikationen, orales Zink mit moderater antientzündlicher Wirkung [28]. In den letzten Jahren wurden zudem eine Bestrahlung mit Blaulicht/Rotlicht, verschiedene Laser sowie die photodynamische Therapie zur Behandlung der Akne eingesetzt [29].

\section{Schlussbemerkung}

$\nabla$

Bei Kenntnis der relevanten pathophysiologischen Faktoren ist eine gute Beratung des Patienten sowie die sinnvolle Auswahl geeigneter Therapiemethoden für jeden Akne-Typ möglich. Die erfolgreiche Therapie ist das beste Mittel zur Besserung der Lebensqualität des Patienten und zur Vermeidung von Narben. 


\section{Abstract}

\section{Pathogenesis and Therapy of Acne: Current Aspects}

Acne vulgaris is a disease with a prevalence of up to $80 \%$ in teenagers. Genetic factors are very important. The disease is caused by a complex interplay of different factors (seborrhoea, cornification disorder, microbial colonisation with Propionibacterium acnes and inflammation). Inflammatory reactions are involved in the initiation of early acne-lesions. Depending on clinical manifestations, the different factors can be treated effectively: cornification disorders with topical retinoids and benzoylperoxide (BPO), bacterial colonisation with antibiotics and BPO, inflammation with antibiotics, seborrhoea with antiandrogens and all factors by isotretinoine. In the last years it was shown that combination therapies proved to be more effective than monotherapies, such as systemic antibiotics and topical retinoids or BPO/ antibiotic combinations. Retinoids are the therapy of choice for maintentance therapy. With these approaches, acne is treated effectively.

\section{Literatur}

1 Rzany B, Kahl C. Epidemiologie der Acne vulgaris. J Dtsch Dermatol Ges 2006; 4: 8-9

2 Jeremy AH, Holland DB, Roberts SG, Thomson KF, Cunliffe WJ. Inflammatory events are involved in acne lesion initiation. J Invest Dermatol 2003; 121: 20-27

3 Moss C. Genetic control of sebum excretion and acne - a twin study. $\mathrm{Br}$ J Dermatol 1989; 121: 144-145

4 Goulden V, Clark SM, Cunliffe WJ. Post-adolescent acne: a review of clinical features. Br J Dermatol 1997; 136: 66 - 70

5 Goulden V, McGeown CH, Cunliffe WJ. The familial risk of adult acne: a comparison between first-degree relatives of affected and unaffected individuals. Br J Dermatol 1999; 141: 297 - 300

6 Herane MI, Ando I. Acne in infancy and acne genetics. Dermatology 2003; 206: $24-28$

7 Blanche H, Vexiau P, Clauin S, Le Gall I, Fiet J, Mornet E, Dausset J, Bellanne-Chantelot $C$. Exhaustive screening of the 21-hydroxylase gene in a population of hyperandrogenic women. Human Genetics 1997; 101: $56-60$

8 Degitz K, Placzek M, Arnold B, Schmidt H, Plewig G. Congenital adrenal hyperplasia and acne in male patients. Br J Dermatol 2005; 148: $1263-1266$

9 Placzek M, Arnold B, Schmidt H, Gaube S, Keller E, Plewig G, Degitz K. Elevated 17-hydroxyprogesterone serum values in male patients with acne. J Am Acad Dermatol 2005; 53: 955 -958

10 Harde V, Muller M, Sippell WG, Schwarz T, Folster-Holst R. „Acne infantum" bei adrenogenitalem Syndrom als Folge eines 11-beta Hydroxylasemangels. J Dtsch Dermatol Ges 2006; 4: 654-657

11 Zouboulis CC. Moderne Aspekte der Aknepathogenese. Akt Dermatologie 2006; 32: $296-302$

12 Ingham E, Eady EA, Goodwin CE, Cove JH, Cunliffe WJ. Pro-inflammatory levels of interleukin-1 alpha-like bioactivity are present in the majority of open comedones in acne vulgaris. J Invest Dermatol 1992; 98: 895-901
13 Guy R, Green M, Kealey T. Modelling of acne in vitro. J Invest Dermatol 1996; 106: 176- 182

14 Pawin H, Beylot C, Chivot M, Faure M, Poli F, Revuz J, Dreno B. Physiopathology of acne vulgaris: recent data, new understanding of the treatments. Eur J Dermatol 2004; 14: 4 - 12

15 Zouboulis CC, Eady AE, Philpott M, Goldsmith LA, Orfanos C, Cunlliffe W, Rosenfield $R$. What is the pathogenesis of acne? Exp Dermatol 2005; 14: $143-152$

16 Mallon E, Newton JN, Klassen A, Stewart-Brown SL, Ryan TJ, Finlay AY. The quality of life in acne: a comparison with general medical conditions using generic questionnaires. Br J Dermatol 1999; 140: 672 -676 17 Cunliffe W. Akne. Stuttgart: Hippokrates, 1993

18 Ozolins M, Eady AE, Avery AJ, Cunliffe WJ, Li Wan Po A, O'Neill C, Simpson NB, Walters CE, Carnegie E, Lewis JB, Dada J, Haynes M, Williams K, Williams HC. Comparison of five antimicrobial regimens for treatment of mild to moderate inflammatory facial acne vulgaris in the community: a randomised controlled trial. Lancet 2004; 364: 2188-2195

19 Thielitz A, Gollnick H. Systemische Aknetherapie. J Dtsch Dermatol Ges 2005; 3: $366-378$

20 Leyden J, Thiboutot DM, Shalita AR, Webster G, Washenik K, Strober BE, Shupack J. Comparison of tazarotene and minocycline maintenance therapies in acne vulgaris: a multicenter, double-blind, randomized, parallel-group study. Arch Dermatol 2006; 142: 605-612

21 Thiboutot DM, Shalita AR, Yamauchi PS, Dawson C, Kerrouche N, Arsonnaud S, Kang S. Adapalene gel, $0.1 \%$, as maintenance therapy for acne vulgaris: a randomized, controlled, investigator-blind follow-up of a recent combination study. Arch Dermatol 2006; 142: 597-602

22 Lookingbill DP, Chalker DK, Lindholm JS, Katz HI, Kempers SE, Huerter CJ, Swinehart JM, Schelling DJ, Klauda HC. Treatment of acne with a combination clindamycin/benzoyl peroxide gel compared with clindamycin gel, benzoyl peroxide gel and vehicle gel: combined results of two double-blind investigations. J Am Acad Dermatol 1997; 37: 590 - 595

23 Worret WI, Fluhr J. Topische Therapie mit Benzoylperoxid, Antibiotika und Azelainsäure bei der Akne. J Dtsch Dermatol Ges 2006; 4: 293 300

24 Ochsendorf F. Systemische Antibiotika zur Therapie der Akne. J Dtsch Dermatol Ges 2006; 4: 828-841

25 Ochsendorf $F$. Clinical relevance of antibiotic resistance in acne. G Ital Dermatol Venereol 2006; 141: 183 - 186

26 Zouboulis CC. Therapie der Akne mit Antiandrogenen - eine Evidenzbasierte Übersicht. J Dtsch Dermatol Ges 2003; 1: 535-546

27 Layton AM, Dreno B, Gollnick HP, Zouboulis CC. A review of the European Directive for prescribing systemic isotretinoin for acne vulgaris. J Eur Acad Dermatol Venereol. 2006; 20: 773 - 776

28 Degitz K, Plewig G. Ergänzende Verfahren in der Aknetherapie. J Dtsch Dermatol Ges 2005; 3: 92 - 96

29 Borelli C, Merk K, Plewig G, Degitz K. Licht, Laser und PDT-Therapie für Akne. Hautarzt 2005; 56: 1027-1032

30 Friedman GD. Twin studies of disease heritability based on medical records: application to acne vulgaris. Acta Genet Med Gemellol 1984; 33: $487-495$

31 Walton S, Wyatt EH, Cunliffe WJ. Genetic control of sebum excretion and acne - a twin study. Br J Dermatol 1988; 118: 393 - 396

32 Cunliffe WJ. Natural history of acne. In: Marks R (ed). Acne and related disorders. London: M. Dunitz, 1989: 4-6

33 Bataille V, Snieder H, MacGregor AJ, Sasieni P, Spector TD. The influence of genetics and environmental factors in the pathogenesis of acne: a twin study of acne in women. J Invest Dermatol 2002; 119: 1317 1322

34 Evans DM, Kirk KM, Nyholt DR, Novac C, Martin NG. Teenage acne is influenced by genetic factors. Br J Dermatol 2005; 152: 579- 581 